\title{
Bilateral neglected Blount's disease in an adult patient: a rare case report
}

\author{
Rahul Temani $^{1}$ *, Hemeshwar Harshvardhan ${ }^{1}$, Ashwini Kumar Khicher ${ }^{2}$, Priyanka Bansal ${ }^{3}$
}

\author{
${ }^{1}$ Department of Orthopaedics, J.L.N. Medical College, Jaipur, Rajasthan, India \\ ${ }^{2}$ Department of Orthopaedics, S.M.S. Medical College, Jaipur, Rajasthan, India \\ ${ }^{3}$ Department of Obstetrician-Gynecologist, Pt. B.D. Sharma, P.G.I.M.S, Rohtak, Haryana, India
}

Received: 29 August 2019

Revised: 12 October 2019

Accepted: 14 October 2019

\author{
*Correspondence: \\ Dr. Rahul Temani, \\ E-mail: temsa08@gmail.com
}

Copyright: () the author(s), publisher and licensee Medip Academy. This is an open-access article distributed under the terms of the Creative Commons Attribution Non-Commercial License, which permits unrestricted non-commercial use, distribution, and reproduction in any medium, provided the original work is properly cited.

\begin{abstract}
Blount's disease is more common in Afro-Caribbean, obese, female child. Early walking have been associated with the disease process. Adult patients with Blount's disease who received no treatment previously, are rarely reported across the other parts of the world and hence less discussed. The treatment of neglected cases of Blount's disease often requires correction of varus deformity, internal tibial torsion and medial plateau depression. We are reporting one such patient seen at our hospital. A skeletally mature, obese (BMI 43.2), male patient with bilateral tibia vara was operated for hemi-plateau elevation combined with a valgus osteotomy. Fixation was achieved with plate and staples. Surgical correction of one such patient is technically demanding and more complicated. If left untreated, the main risk is premature osteoarthritis.
\end{abstract}

Keywords: Blount's disease, Tibia vara, Adult patient, Osteotomy

\section{INTRODUCTION}

Tibia vara or Blount's disease was the first described by Erlacher in 1922 and detailed description was given by Walter Putnam Blount's in 1937. ${ }^{1}$ There are three types of Blount's diseasein viz., infantile-early onset below 4 years of age; juvenile-late onset after 4 years of age (4 years-10 years) and adolescent-late onset after 10 years of age. ${ }^{2}$ Blount's disease is a developmental condition characterized by disordered endochondral ossification of the medial part of the proximal tibial physis resulting in multiplanar deformities of the lower limb. ${ }^{3}$

Blount's disease was once termed Tibia vara, implying the deformity occurred solely in the frontal plane. ${ }^{3}$ It is now believed that many other deformities accompanied tibial varus in this disease. ${ }^{4,5}$ Proximal tibial procurvatum and internal torsion are among the other common deformities found in Blount's disease ${ }^{4}$. Compensatory valgus deformity of the femur, once considered common in Blount's disease, has been proven vice versa. ${ }^{3}$ Adding to the contradiction, distal femoral varus takes portion in causing genu varum in late onset-disease. ${ }^{5}$ Nevertheless, the exact pathophysiology of Blount's disease remains an enigma.

A number of risk factors such as ethnicity (more common in Afro-Caribbean children), sex (female>male), obesity with increased mechanical stress and early walking have been proposed to contribute to the disease process. Langenskiold and Riska proposed elevating the medial plateau for cases of infantile Blount's disease that presented late. ${ }^{6}$ The indication for this additional procedure is the significant incongruity of the joint surface due to depression of the medial part of the tibial plateau. $^{7}$ In addition to the medial plateau elevation, the 
varus of the tibia is now corrected by a valgus tibia osteotomy, either simultaneously or in a separate operation. The term "double elevating osteotomy" was proposed by Gregosiewicz et al who used the bony wedge removed from the tibial valgus osteotomy to support the medial plateau elevation. ${ }^{8}$

\section{CASE REPORT}

A 20 year old male patient presented with deformity in both the legs and altered walking pattern. His immediate postnatal history and developmental milestones (language, social and motor) were normal. There was no history suggestive of trauma or osteomyelitis. There was no relevant family history.

He was obese (weight: $102 \mathrm{~kg}$; height: $168 \mathrm{~cm}$; body mass index: $36.1 \mathrm{~kg} / \mathrm{m}^{2}$, obese BMI) with no stigmata of hypercortisolism/hypothyroidism or Albright's hereditary osteodystrophy. Examination revealed nontender bony prominence over medial tibial condyles, 25 degrees of varus deformity in both the knees. There was 20 degrees of internal tibial torsion bilaterally. There was no tenderness around the knee and no ligamentous laxity. Full range of motion was present in both hips and knees. No limb length discrepancy was present. On a scanogram (Figure 2), metaphyseo-diaphyseal angle was 25 degrees bilaterally, tibio-femoral angle was 25 degrees on both sides and the angle of medial tibial plateu depression was 40 degrees on both sides. Baseline investigations including complete blood count, liver functions, renal functions, electrolytes and arterial blood gas analysis were normal. Serum calcium (albumin corrected value $9.5 \mathrm{mg} / \mathrm{dL})$, phosphorous $(4.8 \mathrm{mg} / \mathrm{dl}), \quad$ alkaline phosphatase, 25-OH-D (31 ng/ml) and intact parathyroid hormone $(47 \mathrm{pg} / \mathrm{ml})$ were also within age-specific reference ranges. Diagnosis of Blount's disease was made on the basis of the clinical and radiological findings. Patient was operated for hemi-plateau elevation combined with a valgus osteotomy. Osteotomy was stabilized with plate and staples and above knee plaster cast was applied for 6 weeks.

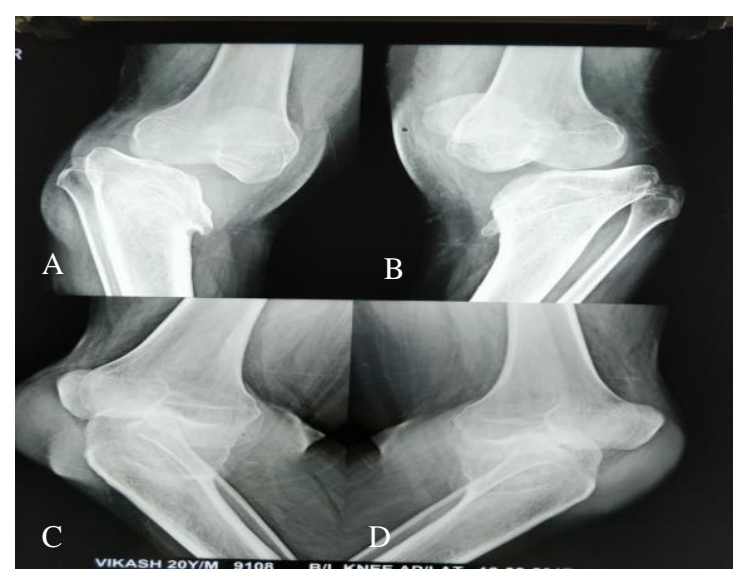

Figure 1 (A-D): Bilateral Blount's disease in a skeletally mature patient.

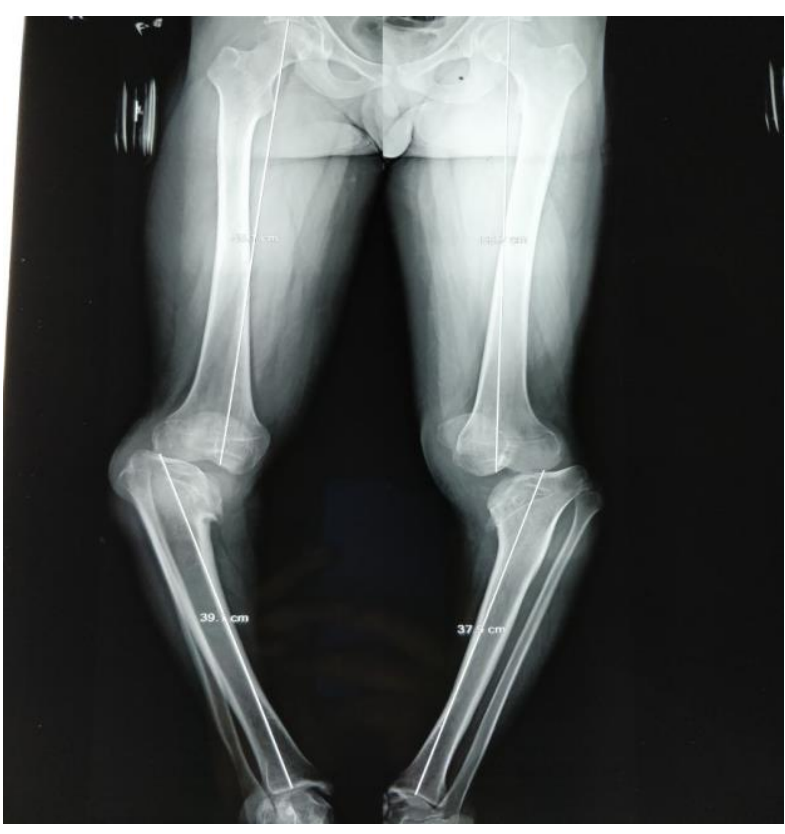

Figure 2: Bilateral metaphyseo-diaphyseal angle and tibio-femoral angle to be $\mathbf{2 5}$ degree each and the angle of medial tibial plateau depression of $\mathbf{4 0}$ degrees on both sides.

\section{Surgical technique}

Under general anesthesia with the use of tourniquet (Figure 3), a mid to distal fibular osteotomy $(1-2 \mathrm{~cm}$ ) was made through a lateral incision. Both tibial osteotomies were performed through the same anteromedial curved in order to avoid recurrences incision. A wedge shaped osteotomy was initially performed about $5 \mathrm{~cm}$ distally of the tibial joint line, below the tibial tuberosity. A bony wedge was removed for correcting the varus and the internal torsion of the tibia. Overcorrection up to 7 degrees of valgus was addressed for reducing the pressure of the medial physic. Fixation was achieved by staples and plate. Second osteotomy for the medial plateau elevation was made just below the "beak" that is formed under image intensifier control (Figure 4). A wide chisel was used to target the indercondylar area without crossing the articular cartilage. Special care was taken for the cut to be parallel to the joint line. During osteotomy, the knee was in flexion for minimizing the risk of provoking neurovascular damage. Thus, a fracture was provoked in the non-weight-bearing indercondylar area and the medial tibial plateau was mobilized. Thereafter, both the bony wedge removed from the first osteotomy and the excised fibula was used as a strut for supporting the new medial plateau position with respect to the continuity of the articular surface (Figure 5). Eventually, the layers of surgical wounds were closed, and above knee cast (plaster of Paris) is used for immobilization. Care was taken to avoid closing the fascia too tightly for the prevention of compartment syndrome. 


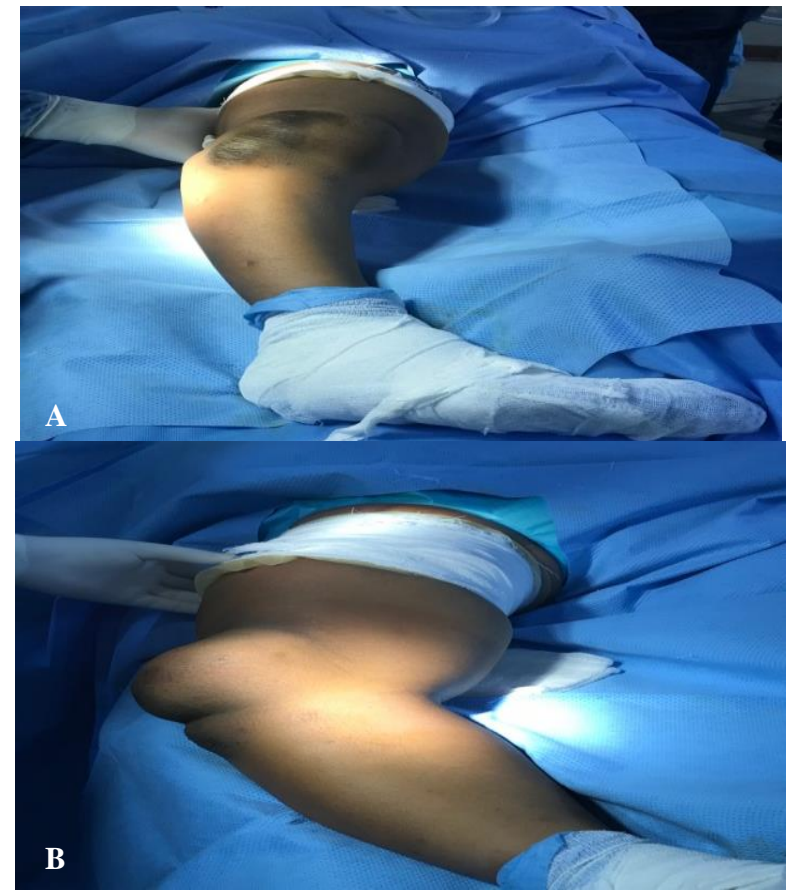

Figure 3 (A and B): Pre-operative image.

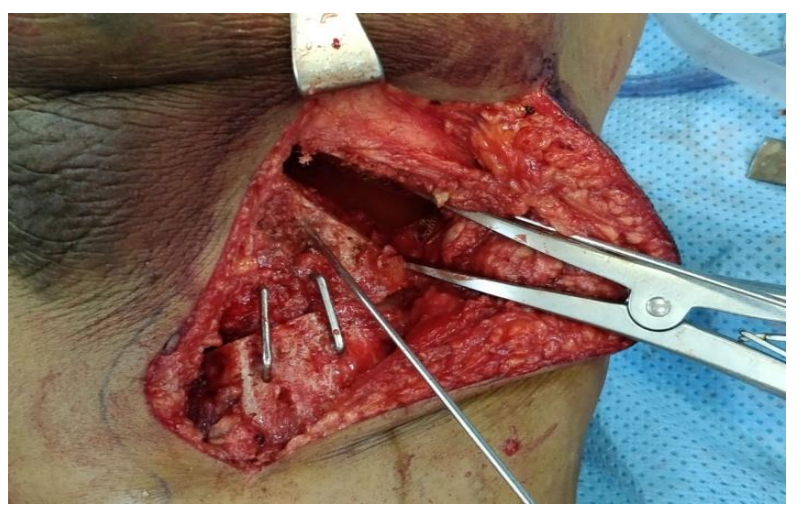

Figure 4: Intra-operative image of the osteotomy for the medial plateau elevation.

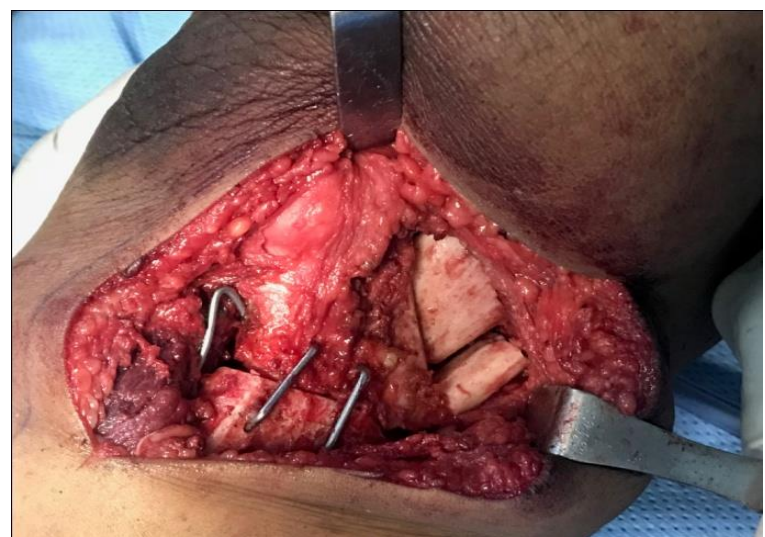

Figure 5: Intra-operative image of the bony wedge removed from the first osteotomy and the excised fibula were used as a strut for supporting the new medial plateau position.

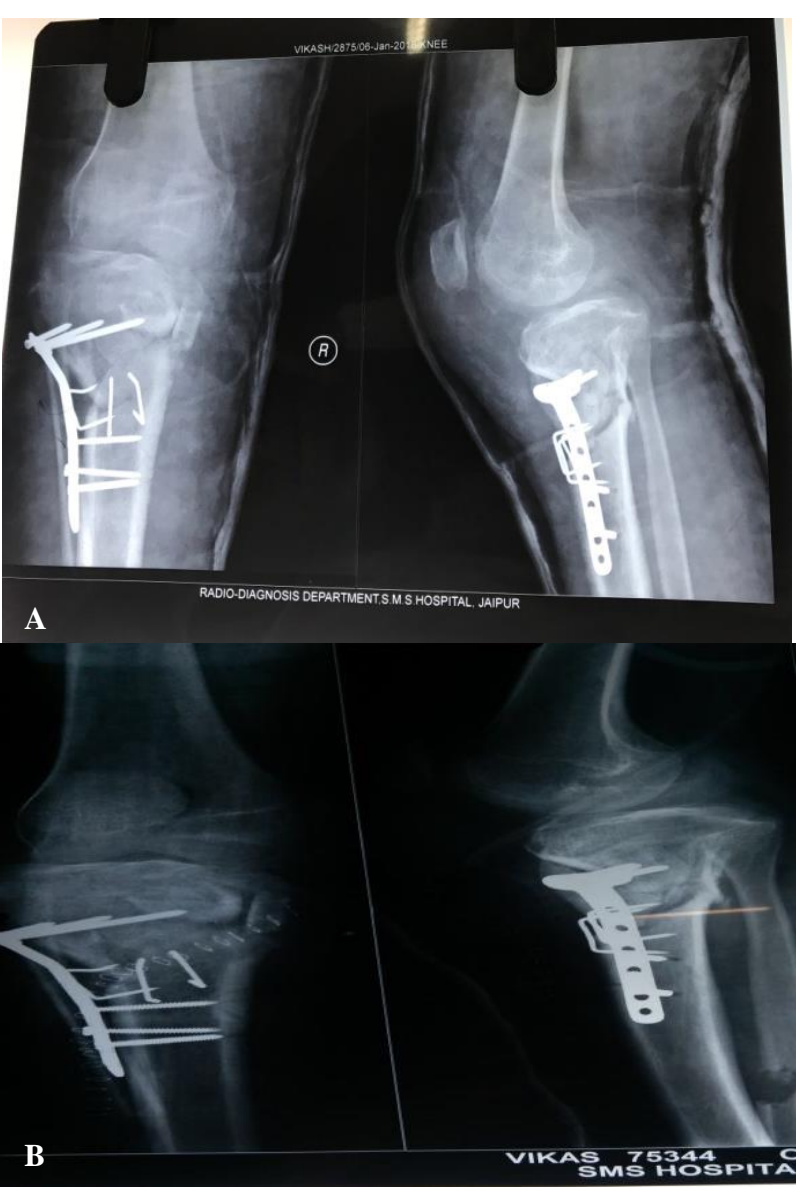

Figure 6 (A and B): Immediate post-operative X-ray.

\section{DISCUSSION}

Blount's disease is commonly seen in African-American lineage and rarely, cases are reported from other parts of world. The treatment of neglected cases of Blount's disease requires both varus correction and medial plateau elevation. Technically, it is a very difficult and relatively high-risk operation. The extra care should be taken intra and post operatively to avoid injury of the adjacent neurovascular structures that could provoke serious complications. Despite the demanding procedure, with this technique the deformity is corrected and the articular surface is restored (Figure 6).

The Ilizarov ring fixator, the Taylor Spatial Frame, and a Multi-Axial Correction external fixation system are some other modern surgical options. ${ }^{9-11}$ These systems enable gradual correction, weight bearing throughout the entire period of treatment and the simultaneous correction of multiplane deformities and leg length discrepancy. ${ }^{10}$

However, as Jones et al report they are more complicated and time consuming surgeries, they depend on the cooperation of the patients, attention must be paid to avoiding premature consolidation of the proximal osteotomy and daily care is required to avoid pin-site infection. ${ }^{9}$ 


\section{CONCLUSION}

Adult patients with Blount's disease who receive no treatment during childhood can still be seen in under resourced countries. Treatment of these patients is very challenging. Only a handful of studies have assessed the outcomes of Blount's disease in adulthood. In adulthood, if left untreated, the main risk is premature osteoarthritis due to a combination of complex angle abnormalities and of severe obesity. In this situation, total knee arthroplasty (TKA) is particularly challenging.

Funding: No funding sources Conflict of interest: None declared

Ethical approval: Not required

\section{REFERENCES}

1. Blount's WP. Tibia vara Osteochondrosis deformans tibiae. $\mathbf{J}$ Bone Joint Surg (Am). 1937;19(1):1-29.

2. Langenskiold A. Tibia Vara : a critical review. Clin Orthop Relat Res. 1989;246:195-207.

3. Sabharwal S. Blount's disease. J Bone Jt Surg Am. 2009;91:1758-76.

4. Sabharwal S, Lee J, Zhao C. Multiplanar deformity analysis of untreated Blount's disease. J Pediatr Orthop. 2007;27:260-5.

5. Gordon JE, King DJ, Luhmann SJ, Dobbs MB, Schoenecker PL. Femoral deformity in tibia vara. J Bone Jt Surg Am. 2006;88:380-6.
6. Langenskio"ld A, Riska EB. Tibia vara (osteochondrosis deformans tibiae): a survey of seventy-one cases. J Bone Joint Surg Am. 1964;46:1405-20.

7. Herring JA. Tachdjian's pediatric orthopaedics, Volume 4. Saunders Elsevier, Philadelphia; 2008.

8. Gregosiewicz A, Wosko I, Kandzierski G, Drabik Z. Double-elevating osteotomy of tibiae in the treatment of severe cases of Blount's disease. J Pediatr Orthop 1989;9:178-81.

9. Jones S, Hosalkar HS, Hill RA, Hartley J. Relapsed infantile Blount's disease treated by hemiplateau elevation using the Ilizarov frame. J Bone Joint Surg Br. 2003;85:565-571.

10. Feldman DS, Madan SS, Ruchelsman DE, Sala DA, Lehman WB. Accuracy of correction of tibia vara: acute versus gradual correction. J Pediatr Orthop. 200626:794-8.

11. Pandya NK, Clarke SE, McCarthy JJ, Horn BD, Hosalkar HS. Correction of Blount's disease by a multi-axial external fixation system. J Child Orthop. 2009;3(4):291-9.

Cite this article as: Temani R, Harshvardhan $\mathrm{H}$, Khicher AK, Bansal P. Bilateral neglected Blount's disease in an adult patient: a rare case report. Int $\mathbf{J}$ Res Orthop 2019;5:1215-8. 